\title{
O controle da raiva: eficácia do treino cognitivo na doença arterial coronariana'
}

\author{
Anger control: the effectiveness of cognitive \\ anger control training in coronary artery disease
}

\author{
Marilda Emmanuel Novaes LIPP2,3 \\ Cláudio PINHO ${ }^{4}$ \\ Ana Carolina CASTELLI ${ }^{3}$ \\ Micheli Gomes dos SANTOS 5 \\ Angela Tamey FUJITA ${ }^{6}$
}

\begin{abstract}
Resumo
Este estudo teve por objetivos averiguar se o treino cognitivo de controle da raiva é eficaz na redução da reatividade cardiovascular de pacientes diagnosticados com doença arterial coronariana, avaliar seu impacto na redução da raiva e no nível de stress e analisar se há correlação significativa entre a raiva e a magnitude na reatividade cardiovascular em momentos de stress experimental. Vinte e nove adultos com doença arterial coronariana participaram da pesquisa. A reatividade cardiovascular foi aferida, antes e após o tratamento em grupo para a raiva, em sessões de role-play representando situações de stress interpessoal. Resultados mostraram que o tratamento reduziu significativamente os índices de raiva para dentro, raiva estado, raiva traço, expressão, reação e controle da raiva e sintomatologia do stress. A magnitude da reatividade da pressão arterial sistólica correlacionou-se com os índices de raiva temperamento. Verificou-se uma redução significativa na reatividade da pressão arterial sistólica na terceira avaliação, realizada seis meses após o treino cognitivo de controle da raiva.
\end{abstract}

Unitermos: Doença arterial coronariana. Raiva. Stress.

\begin{abstract}
The objectives of this study were twofold: to test the effectiveness of Cognitive Anger Control Training in the reduction of cardiovascular reactivity during periods of stress in individuals with coronary artery disease, and to investigate the link between levels of anger and the extent of cardiovascular reactivity at times of stress. Twenty-nine adults suffering from coronary artery disease took part in the study. Cardiovascular reactivity was measured both before and after group treatment for anger, in role-play sessions representing situations of interpersonal stress.

$\nabla \nabla \nabla \nabla$

1 Apoio: Conselho Nacional de Desenvolvimento Cientifico e Tecnológico. Bolsa de Produtividade Processo 301580/2005-7 para M.E.N. LIPP.

2 Pontifícia Universidade Católica de Campinas, Centro de Ciências da Vida, Laboratório de Estudos Psicofisiológicos do Stress. Campinas, SP, Brasil.

3 Pontifícia Universidade Católica de Campinas, Centro de Ciências da Vida, Programa de Pós-Graduação em Psicologia. Av. John Boyd Dunlop, s/n., Prédio Administrativo, Jd. Ipaussurama, 13060-904, Campinas, SP, Brasil. Correspondência para/Correspondence to: M.E.N. LIPP.

- Pontifícia Universidade Católica de Campinas, Centro de Ciência da Vida, Faculdade de Médicina, Disciplina de Cardiologia. Campinas, SP, Brasil.

5 Pontifícia Universidade Católica de Campinas, Centro de Ciências da Vida, Laboratório de Estudos Psicofisiológicos do Stress, Bolsista Apoio Técnico: Conselho Nacional de Desenvolvimento Cientifico e Tecnológico.

- Pontifícia Universidade Católica de Campinas, Centro de Ciências da Vida, Laboratório de Estudos Psicofisiológicos do Stress, Bolsista Iniciação Científica, Conselho Nacional de Desenvolvimento Cientifico eTecnológico.
\end{abstract}


The findings show that the treatment significantly reduced the indices of internal anger, state anger, trait anger, the expression, reaction and control of anger and level of stress. It was also found that systolic blood pressure reactivity had a significant correlation with the indices of temperamental anger and that a significant reduction was seen in systolic blood pressure reactivity in the third evaluation, conducted six months after treatment.

Uniterms: Coronary artery disease. Anger. Stress.

Trabalhos publicados em vários países revelam o interesse de pesquisadores pela busca de variáveis associadas à doença arterial coronariana, tais como: Anand et al. (2008), na Inglaterra; Schwartz et al. (2003), nos Estados Unidos; Alarcón e Ramírez Vallejo (2006), na Colômbia; Benet Rodríguez, Apollinaire Pinnini, Torres Ros e Peraza Pons (2003), na Espanha; Godoy et al. (2007) e Lipp, Frare e Santos (2007), no Brasil. Este interesse advém da compreensão de que a doença cardiovascular é a principal causa de morte no mundo, sendo sozinha responsável por 30\% de todas as mortes (World Heatlh Organization, 2007). O fato de que os fatores de risco mais conhecidos explicam apenas aproximadamente dois terços dos casos de Doença Arterial Coronariana (DAC) tem estimulado um interesse cada vez maior pela identificação de uma possível associação entre fatores psicológicos, hipertensão e DAC (Strike \& Steptoe, 2004).

Devido ao fato de a hipertensão essencial ser um fator de risco pronunciado para a doença coronariana, o interesse no estudo dos fatores etiopatogênicos envolvidos tem crescido acentuadamente; dentre eles, as possíveis variáveis psicológicas e o stress (Dimsdale, 2008; Sparrenberger, Moreira \& Caneppele, 2004). Estudos remetem à identificação da raiva como elemento presente na ontogênese da reação do stress e da hipertensão arterial (Spielberger \& Moscoso, 1996). O stress pode, por outro lado, gerar raiva e aumentos da reatividade cardiovascular, como demonstrado por García-León, Reyes del Paso, Robles e Vila (2003), e Lavoie, Miller, Conway e Fleet (2001). Deste modo, é importante enfatizar o caráter recíproco da díade stress-raiva, em que o stress prévio pode levar à raiva e a raiva, por outro lado, pode também constituir fonte poderosa de stress.

Os resultados de estudos clínicos são consistentes ao descreverem pacientes infartados como sujeitos a altos níveis de raiva (Larkin \& Zayfert, 2004). Mittleman et al. (1995), estudando 1623 pacientes que haviam sofrido infarto até quatro dias antes, mostraram uma alta relação entre episódios de raiva nas últimas horas e o infarto do miocárdio. Não se conhece pesquisas especificamente objetivando averiguar se o tratamento da raiva e, consequentemente, do stress emocional, reduz o risco de infarto por meio da diminuição da reatividade cardiovascular, à qual se tem atribuído uma função significativa na neurogenética da hipertensão (Carvalho \& Krieger, 2003), das doenças coronarianas (Lovallo \& Gerin, 2003) e do acidente vascular cerebral (Gianaros, Jennings, Richard, Derbyshire \&Matthews, 2007).

Estudos da reatividade cardiovascular têm sido realizados em vários laboratórios sob controle experimental para averiguar o mecanismo de ação de aumentos pressóricos desencadeados pelo stress (Fritz, Nagurney \& Helgeson, 2003).

No Brasil, Lipp (2005), em pesquisa com 58 hipertensos, e Lipp, Justo e Melo Gomes (2006), com 80 adultos, mostraram que o hipertenso exibe aumentos de pressão significativos quando submetido a sessões experimentais de stress emocional. Os autores verificaram que variáveis psicológicas, como inassertividade, alexitimia e stress emocional são fatores importantes na determinação da magnitude da reatividade cardiovascular que ocorre em contatos sociais estressantes. A utilização de tarefas estressantes no laboratório, onde se mensura a reatividade cardiovascular com o objetivo de generalizar os dados, justifica-se à medida que a literatura apresenta estudos mostrando a adequação desta inferência. Turner et al. (1994) analisaram 31 estudos publicados até 1993 e concluíram que existe evidência corroborando esta generalização. Steptoe, Cropley e Joekes (2000) alertam que esta generalização é mais previsível quando a tarefa estressante no laboratório não ultrapassa a carga de stress dos conflitos do dia a dia. As tarefas geradoras de stress utilizadas por Lipp et al. (2006) e Lipp et al. (2007) são muito semelhantes ao que se encontra na vida diária. Acredita-se, portanto, que os dados destas pesquisas possam, de fato, ser generalizados para o cotidiano. 
Lipp et al. (2006) demonstraram que o stress é especialmente tóxico quando a pessoa envolvida na interação estressante possui especificamente dois estilos de expressão de raiva: raiva para fora e raiva para dentro. Ambos os estilos podem ser prejudiciais, já que a pressão arterial sofre aumentos consideráveis em momentos de interações estressantes quando essas pessoas ou expressam a raiva de modo explosivo ou inibem excessivamente a expressão da raiva. Estas afirmações podem inicialmente parecer conflitantes (expressar é ruim e inibir também), mas existem evidências na literatura que corroboram os achados desta pesquisa. Por exemplo, Stoney e Engebretson (1994) enfatizaram que expressar a raiva pode aumentar a pressão arterial, enquanto Spielberger et al. (1985) alegam que deixar de expressar a raiva pode acarretar uma série de problemas de saúde, inclusive a hipertensão.

O estudo de Davidson, MacGregor, Stuh, Dixon e MacLean (2000), com 1862 participantes adultos, lança luz sobre estes dados aparentemente conflitantes, ao demonstrar que a expressão construtiva da raiva relaciona-se com pressão arterial basal mais baixa, indicando que a expressão adequada da raiva parece ser a forma mais apropriada no que se refere à manutenção fisiológica da pressão arterial.

Gidron, Davidson e Bata (1999) testaram a eficácia de uma intervenção cognitivo-comportamental no controle da raiva em pacientes coronarianos e verificaram que o nível de hostilidade foi reduzido, bem como a pressão diastólica, que se manteve mais baixa no acompanhamento realizado dois meses após o término da intervenção.

Com base nos achados de Davidson et al. (2000) e Gidron et al. (1999), levantou-se a hipótese de que um trabalho psicológico junto a pessoas com doenças coronarianas e hostis, com um alto nível de raiva para fora, explosiva, permeada com muita agressividade, e pessoas com nível elevado de raiva para dentro, seria eficaz na redução da reatividade cardiovascular em momentos de stress. $\mathrm{O}$ estudo aqui descrito se propôs, portanto, averiguar se o Treino Cognitivo de Controle da Raiva (TCCR) é eficaz na redução da reatividade cardiovascular de pacientes diagnosticados com doença arterial coronariana, avaliar seu impacto na redução da raiva e no nível de stress e analisar se há correlação significativa entre a raiva e a magnitude na reatividade cardiovascular em momentos de stress experimental.

\section{Método}

\section{Participantes}

Participaram do estudo 29 portadores de DAC (vinte mulheres e nove homens) na faixa etária de 35 a 60 anos, com média de 54 anos. Os critérios de inclusão foram: ser portador de DAC; estar estável e sob controle ambulatorial eficaz; não possuir distúrbio psiquiátrico grave; ter tempo disponível para o tratamento, que envolveu 14 visitas ao laboratório. A seleção final dos participantes ocorreu por meio de entrevista psicológica de pacientes atendidos no setor de cardiologia que foram encaminhados pelos cardiologistas do Hospital e Maternidade Celso Pierro (HMCP). Cinco participantes foram reavaliados uma terceira vez, seis meses após a finalização do TCCR. Não houve um critério de seleção dos mesmos; eles foram incluídos na testagem por serem os únicos com disponibilidade de tempo para comparecer ao laboratório para nova avaliação. Muitos dos participantes do grupo haviam se mudado da área ou não tinham como se afastar do emprego durante o dia.

\section{Instrumentos}

Além do termo de consentimento livre e esclarecido aprovado pelo Comitê de Ética da Pontifícia Universidade Católica de Campinas (protocolo 397/05), foram utilizados os seguintes instrumentos:

Para coleta de dados psicológicos: foram utilizados o inventário de Expressão de Raiva como Estado e Traço (STAXI) de Spielberger (1992); o inventário de Sintomas de Stress de Adultos (Lipp, 2000); o manual do TCCR e a cartilha elaborada especialmente para a pesquisa com orientações sobre como controlar a raiva.

O TCCR é baseado nos conceitos comportamental-cognitivos e inclui: a) identificar as cognições (pensamentos avaliativos da situação sendo vivenciada) e perceber as sensações físicas e emocionais que antecedem a expressão da raiva; b) utilizar técnicas cognitivo-comportamentais de reestruturação cognitiva e de parada de pensamento; c) usar respiração profunda e relaxamento para redução da excitabilidade fisiológica gerada pela ação energética da raiva; d) usar "ação responsável", ou seja, emissão de um compor- 
tamento que resolva, ou reduza, a situação geradora da raiva, por meio de um comportamento adequado ao evento presente.

Para a coleta de dados fisiológicos: foi utilizado um Monitor de Pressão Arterial (FINAPRESS) (Ohmeda, modelo 2300, Denver, Colorado), que mede a pressão arterial e a frequência cardíaca continuamente, de modo não invasivo, através de cuff com um fotopletismógrafo, que é colocado ao redor do dedo médio da mão não dominante do paciente. Ao ser conectado ao computador, fornece a listagem das medidas registradas, com médias de 10 em 10 segundos.

Utilizou-se também uma câmera de vídeo, um gravador e duas fitas contendo as cenas que geram stress social. Cada fita inclui sete cenas que, em pesquisas anteriores (Lipp, 2005), foram utilizadas para criarem stress social nos participantes. As cenas se referem a situações passíveis de ocorrerem na vida real. Uma fita foi utilizada na avaliação pré-tratamento e a outra no pós-tratamento. Testagens anteriores mostraram que elas são equivalentes no nível de stress que eliciam. A ordem de apresentação das fitas foi de bloco randômico, para garantir que a ordem não afetasse o resultado.

O estudo foi realizado no Laboratório de Estudos Psicofisiológicos do Stress (LEPS), Pontifícia Universidade Católica de Campinas.

Quatro psicólogas e quatro cardiologistas colaboraram neste estudo.

\section{Procedimentos}

Todos os participantes passaram por avaliações médicas e psicológicas, em dois momentos distintos: no início do estudo e após o tratamento, e uma subamostra de cinco pacientes passou por uma terceira avaliação seis meses após a finalização do TCCR.

Após a primeira avaliação médica e assinatura do termo de consentimento, os voluntários eram entrevistados por uma psicóloga que procedia à coleta dos dados. Subsequentemente era agendada a primeira testagem experimental. Nesta sessão, o participante sentava em uma cadeira confortável e uma assistente de pesquisa colocava o manguito em seu dedo. Nos primeiros dez minutos, o participante permanecia sozinho na sala, enquanto sua pressão era aferida. A assistente de pesquisa, então, voltava juntamente com outra psicóloga; a primeira fazia os registros no computador e a segunda interagia com o participante após este ouvir cada descrição das cenas. O participante era livre para responder como desejasse, sem nenhuma interferência sobre como proceder. Depois das cenas de stress emocional as assistentes de pesquisa saíam da sala e o participante ficava por mais dez minutos. Após esta sessão, era marcada a data para início do tratamento para a raiva. As sessões terapêuticas foram realizadas em cinco grupos de seis participantes, em média. A pesquisadora principal e uma coterapeuta estavam sempre presentes nas sessões dos grupos, que eram gravadas.

\section{Resultados e Discussão}

\section{Método de análise dos dados coletados}

Para analisar a evolução das variáveis categóricas entre as duas avaliações foi utilizado o teste de McNemar. Para analisar a evolução das variáveis numéricas medidas uma única vez em cada avaliação foi utilizado o teste de Wilcoxon para amostras relacionadas (Signed Rank Test) e, para verificar a evolução da reatividade cardiovascular entre as duas avaliações, considerando as sete cenas (com instrução e resposta) e a linha de base 2, foram calculadas as médias gerais em cada avaliação (15 pontos de medida), assim como as médias das cenas de instrução (7 pontos de medida) e das cenas de resposta (7 pontos de medida), e utilizado o teste de Wilcoxon para amostras relacionadas (Signed Rank Test). Para analisar a relação dos vários parâmetros foram calculados os deltas percentuais entre as duas avaliações e utilizado o coeficiente de correlação de Spearman. 0 nível de significância adotado para os testes estatísticos foi de $5 \%$.

\section{Dados sociodemográficos}

A amostra foi constituída de 69\% de mulheres e 31\% de homens. A predominância do gênero feminino deve-se provavelmente ao fato de que o estudo exigia a presença no LEPS de no mínimo 14 vezes, e nem todos os homens podiam se ausentar do trabalho tantas vezes. 
A maioria da amostra (61\%) tinha idade superior a 49 anos, que é compatível com a faixa etária de prevalência deDAC.

\section{A raiva e suas várias dimensões}

Como esperado com base na literatura da área, verificou-se uma alta incidência de raiva, o que confirma a sugestão de Spielberger e Moscoso (1996) de que a raiva é um fator presente na ontogênese da reação do stress e da hipertensão arterial. Confirmaram-se também os dados de Mittleman et al. (1995), que encontraram uma alta relação entre episódios de raiva nas últimas horas e o infarto do miocárdio.

OTCCR foi eficaz na promoção de mudança em vários aspectos, conforme revelado pela comparação estatística dos dois momentos (antes e pós-tratamento). Cada dimensão desta variável psicológica foi analisada separadamente (Figura 1).

Raiva traço: a diferença nos índices de raiva traço, entre as duas avaliações, foi significativa. Na primeira, o percentual de pessoas acima da média era 55,17\%, e no pós-tratamento passou a ser 34,48\% ( $S=4,50 ; \mathrm{GL}=1$; $p=0,034)$. A mudança observada foi interessante, uma vez que o traço de raiva é considerado um aspecto mais estável do que o estado de raiva, o que mostra a eficácia do TCCR.

Reação de raiva: na primeira testagem, 48,28\% estavam acima da média e, na segunda, este percentual foi reduzido para 31,03\%. Essa diferença é significativa $(S=3,57 ; G L=1 ; p=0,050)$.

Expressão de raiva: a diferença entre os dois momentos na expressão de raiva foi significativa $(S=8,33$;

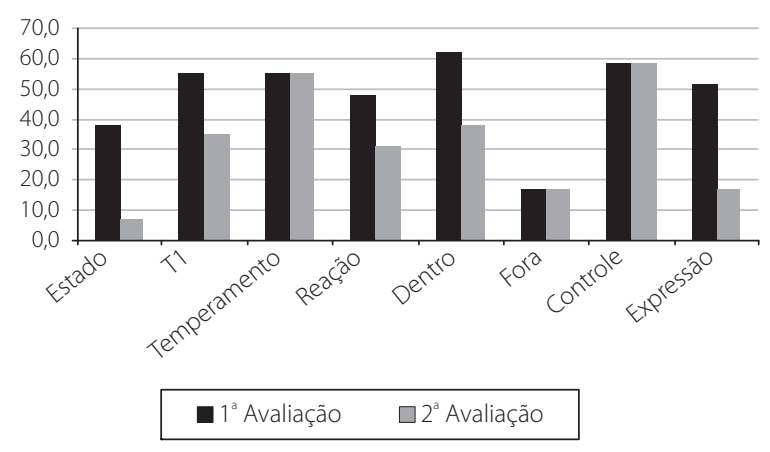

Figura 1. Comparação das várias dimensões da raiva nas duas avaliações.
$\mathrm{GL}=1 ; p=0,004)$, com $51,72 \%$ dos participantes expressando mais raiva no primeiro momento em comparação a 17,24\% deles no segundo. Este dado pode ser compreendido considerando-se que se os participantes sentiam menos raiva (demonstrado pela redução no traço e na reação de raiva), era esperado que também a expressassem menos. O TCCR possui duas áreas de atuação: uma na qual a pessoa adquire uma percepção do mundo ao seu redor menos ameaçadora ou conflituosa e, portanto, a raiva surge com menor frequência; a segunda área se refere à expressão adequada da raiva, em situações nas quais ela é desenvolvida. Há que se entender que a expressão da raiva não significa colocá- la para fora de modo agressivo, mas, como o TCCR enfatizou, é importante que a raiva seja expressa, de modo a promover uma solução para o conflito presente.

Raiva para dentro: verificou-se uma diferença significativa entre o índice de pessoas que usavam o recurso de jogar a raiva para dentro antes e após o tratamento da raiva $(S=5,44 ; G L=1 ; p=0,020)$. Antes, $62,07 \%$ dos participantes lidavam com a raiva colocando-a para dentro. Após o TCCR, este percentual foi reduzido para 37,90\%. Pode-se deduzir que, tendo aprendido a lidar melhor com seus sentimentos de raiva, $62 \%$ dos participantes não utilizavam mais o recurso de colocar a raiva para dentro em situações estressantes.

Raiva para fora: a porcentagem de pessoas na amostra que colocavam para fora, de algum modo, a raiva que sentiam não mudou nas duas avaliações, ficando em 82,76\% ( $S=0,00 ; G L=1 ; p=1,000)$, como também não mudou o índice de controle da raiva, com 58,62\% exercendo um controle acima da média $(S=0,00 ; G L=1$; $p=1,000$ ). Este dado era esperado, pois o TCCR enfatiza a necessidade de se colocar para fora, de modo construtivo, a raiva que se sente, e não reprimi-la, simplesmente. O controle foi também um aspecto muito enfatizado no tratamento, portanto, o resultado obtido era esperado.

Raiva temperamento: Spielberger (1992) postula que a variável temperamento é a mais estável das dimensões da raiva. Os dados da pesquisa aqui descrita dão suporte a esta afirmação, à medida que não houve diferença alguma nas avaliações antes e após o TCCR $(S=0,00 ; G L=1 ; p=1,000)$. Nenhum dos entrevistados mudou neste aspecto, e o percentual deles com raiva 
temperamento acima da média permaneceu 55,17\% no pós-tratamento. Interessante notar que esta foi a única subescala da raiva que não sofreu qualquer alteração em função do TCCR, mostrando que, embora a pessoa possa aprender a controlar e lidar com a raiva, a tendência a senti-la é forte em algumas pessoas.

Raiva estado: na primeira avaliação, 37,93\% encontravam-se com o sentimento de raiva presente. Na segunda avaliação, a porcentagem de pessoas com raiva, no exato momento da entrevista, foi reduzida para $6,90 \%$, o que representa uma diferença significativa $(S=6,23 ; G L=1 ; p=0,013)$ entre os dois momentos, reveladora de maior tranquilidade pós-tratamento.

Analisando apenas as dimensões nas quais ocorreram mudanças, chega-se à conclusão de que o mecanismo pelo qual o TCCR funcionou foi primordialmente na redução do surgimento da raiva. Em vez de simplesmente levar os participantes a exercerem controle do seu comportamento raivoso, o TCCR os capacitou para avaliarem o mundo ao seu redor de modo menos provocador. A percepção mais amena dos eventos da vida, acoplada à ideia do uso de ação responsável na resolução dos problemas do viver, é útil para reduzir as ondas de raiva que, muitas vezes, comprometem o convívio na sociedade e prejudicam a saúde, principalmente de pessoas com DAC.

\section{O stress emocional na amostra}

Na avaliação pré-tratamento, 82,36\% dos participantes tinham sintomas significativos de stress, sendo que 44,43\% estavam na fase intermediária do stress e 37,93\% encontravam-se em fase já patológica: a quase exaustão (Figura 2). Este percentual ultrapassa muito a média do estado de São Paulo, que é de aproximadamente 12\% na fase de quase exaustão (Lipp, 2008). A maior prevalência de sintomas, tanto na primeira como na segunda avaliação, foi de natureza psicológica, o que indica a importância dos aspectos emocionais no funcionamento dos entrevistados. Na segunda avaliação, o número de participantes na fase avançada do stress caiu para 17\%, e o número de pessoas sem stress significativo aumentou para 51,73\%. A diferença foi significativa $(S=7,36 ; G L=1 ; p=0,007)$ (Figura 2).

No que se refere à variável psicológica, verificou510 -se que o tratamento cognitivo-comportamental da

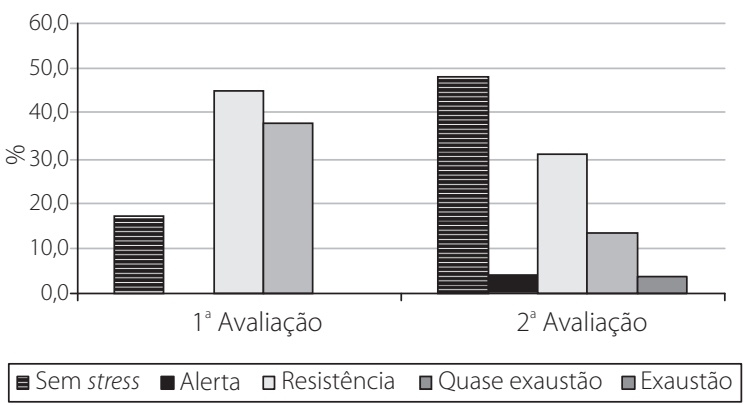

Figura 2. Stress nas duas avaliações.

raiva foi eficaz nas modificações produzidas, tendo havido melhora em como os pacientes reagiam às situações de stress interpessoal. A redução no nível de stress emocional verificada após o tratamento para a raiva indica a relação entre raiva e stress, mostrando que a raiva pode ser considerada um fator precipitante do stress emocional, pelo menos entre pacientes portadores de DAC. Estes dados mostram a importância de se averiguar mais completamente a associação entre fatores psicológicos, hipertensão arterial e DAC, conforme sugerido por Strike e Steptoe (2004).

\section{Medidas cardiovasculares: reatividade cardiovascular no pré e pós-tratamento}

Embora os participantes tenham mostrado redução da reatividade cardiovascular em alguns momentos da segunda sessão experimental, a diferença entre a primeira e a segunda avaliação não foi significativa (Figura 3 e 4). A estabilidade da medida de reatividade cardiovascular registrada nas duas avaliações pode ter sido o produto de outros fatores psicológicos não identificados no estudo. Por exemplo, um trabalho recém-publicado por Salomon, Clift, Karlsdóttir e Rottenberg (2009) revelou que o estado depressivo de pacientes pode influenciar a reatividade cardiovascular em momentos de stress experimental. No estudo aqui relatado a variável depressão não foi avaliada, e poderia estar afetando os resultados no que se refere à reatividade.

\section{Reatividade cardiovascular na terceira avaliação}

A avaliação de follow-up da subamostra de cinco dos participantes da pesquisa que foram chamados seis 


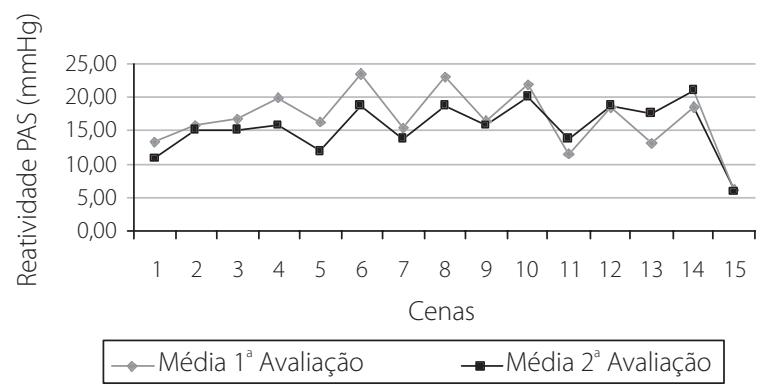

Figura 3. Reatividade da PAS nas duas avaliações em cada momento das sessões experimentais.

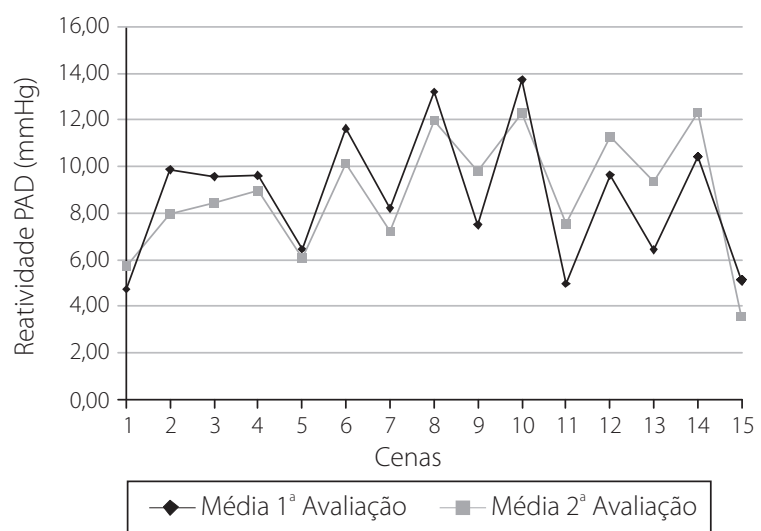

Figura 4. Reatividade da PAD nas duas avaliações em cada momento das sessões.

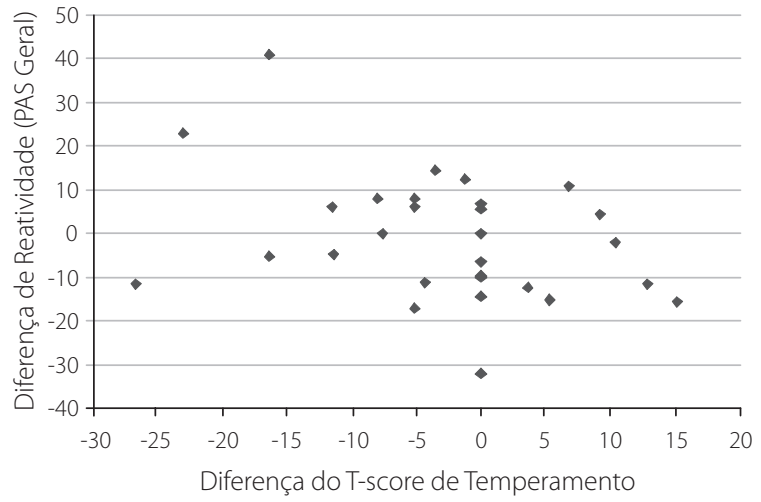

Figura 5. Correlação entre deltas de reatividade da PAS e mudanças nos escores de temperamento da raiva.

meses após a segunda avaliação mostrou que houve uma diferença significativa na reatividade da pressão arterial sistólica entre a segunda avaliação e a terceira, revelando uma redução significativa na pressão arterial dos participantes na última (Teste de Comparações Múltiplas de Turkey-Kramer, $\mathrm{q}=4,823 ; p=0,0037)$. Este dado indica que o TCCR continua a ter efeito com o passar do tempo. É possível que a pessoa que passa por este treino necessite de um tempo maior para processar e assimilar o conteúdo do treinamento. Isto explicaria porque não houve mudança significativa na reatividade cardiovascular logo após o TCCR.

\section{Interação raiva $x$ reatividade cardiovascular}

Para averiguar a possibilidade da existência de uma correlação entre mudanças nos índices de raiva entre as duas avaliações e a diferença em magnitude da reatividade nas duas sessões experimentais, conduziu-se uma análise de correlação entre deltas/diferenças. Para tal, foi calculado o coeficiente de correlação de Spearman entre as diferenças, considerando-se cada tipo de indicador de reatividade (PAS, PAD, Pressão Arterial Média - PAM e Frequência Cardíaca - FC) em função da dimensão da raiva avaliada. Os resultados indicaram uma correlação significativa entre a variação da reatividade da PAS geral e o T - escore de temperamento $(r=-0,3705 ; p=0,0454)$; ou seja, observou-se uma maior redução da reatividade da PAS geral para os que mostraram menor redução do $T$ - escore de temperamento, conforme pode ser observado na Figura 5. A dimensão raiva temperamento foi apontada pelo autor do Inventário de Expressão de Raiva como Estado e Traço utilizado neste estudo para mensurar a raiva (Spielberger, 1992) como a mais inflexível das dimensões da raiva-e, de fato, este foi o aspecto que menos revelou mudança com o tratamento. Assim, a associação negativa entre maior redução em reatividade e menor queda do escore da raiva temperamento foi inesperada. Seria interessante que outros estudos investigassem esta descoberta.

\section{Avaliação médica pós-tratamento}

Todos os participantes passaram por uma avaliação com um cardiologista após o TCCR, que verificou uma estabilidade do quadro clínico, com manutenção da pressão arterial bem controlada e adesão dos pacientes ao tratamento médico indicado. Os cinco 
participantes que constituíram a subamostra chamada para o follow-up apresentaram permanência estável da condição cardiológica.

\section{Considerações Finais}

Os dados indicaram que o TCCR foi eficaz na mudança de quase todos os aspectos psicológicos estudados, pois reduziu o stress e reduziu várias dimensões da raiva de uma amostra de pacientes cardiopatas comprometidos em aspectos emocionais, porém não houve redução significativa da reatividade cardiovascular dos participantes na avaliação fisiológica realizada imediatamente após o tratamento. Houve, no entanto, uma redução significativa da reatividade da pressão arterial sistólica na avaliação realizada no follow-up de seis meses. Uma possível explicação é que o prazo de apenas oito semanas de tratamento não seja suficiente para que mudanças ocorram tão rapidamente na reatividade cardiovascular de pacientes já portadores de doenças coronarianas. Esta população pode necessitar de mais tempo para assimilar e praticar as estratégias de enfrentamento da raiva para que os efeitos fisiológicos levem mais tempo para se manifestar.

Os dados permitem concluir que o TCCR apresenta um modelo teórico-prático para o tratamento de pessoas que tipicamente respondem ao mundo com raiva e que têm pouco controle sobre suas emoções, principalmente as portadoras de DAC. Os resultados sugerem novos estudos para definir se um tempo maior de TCCR poderia modificar a reatividade cardiovascular em benefício do paciente. Considerando-se os dados do estudo de Solomon, Clift, Karlsdóttir e Rottinberg (2009), sugere-se também que a influência da depressão na reatividade cardiovascular em momentos de stress seja estudada em pacientes com DAC. Recomenda-se que novas avaliações enfatizem mudanças em raiva temperamento, que se mostrou mais resistente ao TCCR. Recomenda-se também que uma nova avaliação de follow-up seja realizada com todos os participantes seis meses após o término do TCCR, com o objetivo de verificar se a prática diária das técnicas ensinadas pode influenciar a reatividade durante períodos de stress emocional.

\section{Referências}

Alarcón, R., \& Ramírez Vallejo, E. (2006). Medicina psicosomática en enfermedad cardiovascular: consideraciones clínicas. Revista Colombiana de Psiquiatria 35 (supl.1), $1-15$.

Anand, S. S., Islam, S., Rosengren, A., Franzosi, M. G., Steyn, K., Yusufali, A. H., et al. (2008) On behalf of the INTERHEART Investigators. Risk factors for myocardial infarction in women and men: insights from the INTERHEART study. European Heart Journal, 29 (7), 932-940.

Benet Rodríguez., M., Apollinaire Pinnini, J., Torres Ros, J., \& Peraza Pons, S. (2003). Reactividad cardiovascular y factores de riesgos cardiovasculares en individuos normotensos menores de 40 años. Revista Espanola de Salud Publica, 77 (1), 577-584.

Carvalho, M. H. C., \& Krieger, E. M. (2003). Conceito de significado da hiper-reatividade vascular. Programa de Educação Continuada, 1 (6), 26-30.

Davidson, K., MacGregor, M. W., Stuhr, J., \& Dixon, K. (2000). Constructive anger verbal behavior predicts blood pressure in a population-based sample. Health Psychology, 19 (1), 55-64.

Dimsdale, J. E. (2008). Psychological Stress and Cardiovascular Disease Journal of the American College of Cardiology, 51 (13), 1237-46

Fritz, H. L., Nagurney, A. J., \& Helgeson, V. S. (2003). Social interactions and cardiovascular reactivity during problem disclosure among friends. Personality and Social Psychology Bulletin, 29 ( 6), 713-725.

García-León, A., Reyes del Paso, G. A., Robles, H., \& Vila, J. (2003). Relative effects of harassment, frustration, and task characteristics on cardiovascular reactivity. International Journal of Psychophysiology, 47 (2), 159-173

Gianaros, P. J., Jennings, J., Richard; S., L. K., Derbyshire, S. W.G., \& Matthews, K. A. (2007). Heightened functional neural activation to psychological stress covaries with exaggerated blood pressure reactivity. Hypertension, 49 (1),134-140.

Gidron, Y., Davidson, K., \& Bata, I. (1999). The short-term effects of a hostility-reduction intervention on male coronary heart disease patients. Health Psychology, 18 (4), 416-420.

Godoy, M. F., Lucena, J. M., Miquelin, A. R., Paiva, F. F., Oliveira, D. L. Q., Augustin Junior, J. L., et al. (2007). Mortalidade por doenças cardiovasculares e níveis socioeconômicos na população de São José do Rio Preto, estado de São Paulo, Brasil. Arquivo Brasileiro de Cardiologia, 88 (2), 200-206.

Larkin, K. T., \& Zayfert, C. (2004). Anger management training with mild essential hypertensive patients. Journal of Behavioral Medicine, 19 (5), 415-433.

Lavoie, K. L, Miller, S. B, Conway, M., \& Fleet, R. P. (2001). Anger, negative emotions, and cardiovascularreactivity during interpersonal conflict in women. Journal of Psychosomatic Research, 51(3), 503-512.

Lipp, M. E. N. (2000). Inventario de sintomas de stress para adultos. São Paulo: Casa do Psicólogo. 
Lipp, M. E. N. (2005). Blood pressure reactivity to social stress in an experimental situation. Revista de Ciências Médicas, 14 (4), 317-326.

Lipp, M. E. N. (2008). A dimensão emocional da qualidade de vida. In Ogata \& Marchi (Orgs.), Wellness (pp.51-76). São Paulo: Editora Campus.

Lipp, M. E. N., Frare, A., \& Santos, F. U. S. (2007). Efeitos de variáveis psicológicas na reatividade cardiovascular em momentos de stress emocional. Estudos de Psicologia (Campinas), 24 (2), 161-167.

Lipp, M. E. N., Justo, A. P., \& Mello Gomes, T. M. (2006). Cardiovascular reactivity: differential effect of expressing and inhibiting emotions during moments of interpersonal stress. The Spanish Journal of Psychology, 9 (2), 154-161.

Lovallo, W. R., \& Gerin, W. (2003). Psychophysiological reactivity: mechanisms and pathways to cardiovascular disease. Psychosomatic Medicine, 65 (1), 36-45.

Mittleman, M. A., Maclure, M., Sherwood, J. B. , Mulry , R. P., Tofler, G. H., Jacobs, S.C., et al. (1995). Triggering of acute myocardial infarction onset by episodes of anger . Circulation, 92 (7), 1720-1725.

Schwartz, A. R., Gerin, W., Davidson, K. W., Pickering ,T. G., Brosschot, J. F., Thayer, J. F, et al. (2003). Toward a causal model of cardiovascular responses to stress and the development of cardiovascular disease. Psychosomatic Medicine, 65 (1), 22-35.

Salomon, K., Clift, A., Karlsdóttir, M., \& Rottenberg, J. (2009). Major depressive disorder is associated with attenuated cardiovascular reactivity and impaired recovery among those free of cardiovascular disease. Health Psychology, 28 (2), 157-165.

Sparrenberger, F., Moreira, L.B., \& Caneppele, M. (2004). Associação entre estresse e hipertensão. Revista da Sociedade Brasileira de Hipertensão, 7 ( 3), 96-99.
Spielberger, C. (1992) Inventário de expressão de raiva como estado e raiva. São Paulo: Vector.

Spielberger, C., Johnson, E. H., Russel, S.F., Crane, R. J. Jacobs, G.A., \& Worden, T. J. (1985). The experience and expression of anger: construction and validation of an anger expression scale. In M. A. Chesney \& R. A. Rosenman (Eds.), Anger and hostility in cardiovascular and behavioral disorders (pp. 5-30). New York: Hemisphere.

Spielberger, C., \& Moscoso, M. (1996). Reacciones emocionales del estres: ansiedad y colera. Avances in Psicologia Clinica Latino-Americana, 14, 59-81.

Steptoe A., Cropley, M., \& Joekes, K. (2000). Task demands and the pressures of everyday life: associations between cardiovascular reactivity and work blood pressure and heart rate. Health Psychology, 19 (1), 46-54.

Stoney, C. M., \& Engebretson, T. O. (1994). Anger and hostility: potential mediators of the gender difference in coronary heart disease. In W. Siegman \& T. W. Swmith (Eds.), Anger, hostility and the heart (pp. 215-237). Hilldale, NJ: Erlbaum.

Strike, P. C., \& Steptoe, A. (2004). Psychosocial factors in the development of coronary artery disease. Progress in Cardiovascular Diseases, 46 (4), 337-47.

Turner J. R., Ward, M., Gellman, M., Johnston, D., Light, K., \& van Doornen, L. (1994). The relationship between laboratory and ambulatory cardiovacular activity: current evidence and future directions. Annals of Behavioral Medicine, 16 (1), 12-23.

World Health Organization. (2007). Prevention of cardiovascular disease: guidelines for assessment and management of cardiovascular risk. Washington: WHO Press.

Recebido em: 2/3/2009

Versão final reapresentada em: 27/5/2009

Aprovado em: 29/9/2009 